\title{
A NEW CONTROL STRATEGY FOR A SIGNALIZED TRAFFIC INTERSECTION
}

\author{
Allen R. Stubberud, Xiao-Hua Yu \\ Department of Electrical and Computer Engineering \\ University of California, Irvine \\ Irvine, CA 92697, USA
}

\section{Keywords: Traffic control (transportation), Markov processes, Adaptive control}

\section{ABSTRACT}

A traffic control problem can be formulated as a decision-making problem for a stochastic dynamic system. Optimal traffic signal settings at intersections can minimize the vehicle delay time or the queue length at a stop line. In this paper, a new adaptive control strategy for signalized intersections is developed and tested by simulation. The simulation results show significant improvement over the traditional fully actuated control algorithm, especially for the case of high volume traffic demand.

\section{INTRODUCTION}

Setting signals at intersections to minimize the queue length and/or vehicle delay time is an important goal in traffic management. Typically, traffic signals operate in one of three different control modes at a signalized intersection, namely, pre-timed control, semiactuated control and fully-actuated control [3] [4]. In pre-time control, all the control parameters are fixed and preset. In fully actuated control, both the cycle length and the green time for every phase of the intersection can be varied. At every time step, the controller checks whether an arrival has occurred on any lane of the intersection. If an arrival has occurred, then the phase is given an extension if it has a green indication. If the phase does not have a green, a call is registered for that phase. To determine the signal indication of next phase, all the calls need to be taken into account. The phase sequence is fixed. However, certain phases in the cycle may be skipped if there is no demand detected by detectors. In general, actuated control signals perform better than the pre-timed signals; however, in some complicated cases, e.g., when the traffic demand fluctuates randomly, or when traffic volume approaches the intersection capacity, even fully-actuated control cannot provide a satisfactory solution.

This paper proposes a new and more efficient control strategy based on Markov decision theory. The fundamental work on Markov processes was performed by A. Markov in 1907. By 1950, research on decision problems involving Markov processes had started. Since then, the Markov decision process, or the controlled Markov process, has been studied and applied in many areas, such as physics, chemistry, biology and operations research. A discrete time, stationary, Markov control model is defined on $(\mathrm{X}, \mathrm{A}, \mathrm{P}, \mathrm{R})$ where $\mathrm{X}$, the state space, is a Borel space and every element in the space $\mathrm{x} \in \mathrm{X}$ is called a state; $\mathrm{A}$ is defined as the set of all possible controls (or alternatives); $\mathrm{P}$ is a probability measure space, in which an element $p_{i j}^{k}$ denotes the transition probability from state $\mathrm{i}$ to state $\mathrm{j}$ under alternative $\mathrm{k}$; and $\mathrm{R}$ is a measurable function, also called a one-step reward.

Choosing a particular alternative results in an immediate reward and a transition probability to the next step. The total expected discounted reward over an infinite period of time is defined as:

$$
J \triangleq E\left[\sum_{t=0}^{\infty} \beta^{t} r\left(x_{t}, a_{t}\right)\right]
$$


where $r$ is the one-step transition reward, $\beta$ ( 0 $\leq \beta<1$ ) is the discount factor, and a is the policy. The optimal reward $\mathrm{v}^{*}$, or the supremum (least upper bound) of $\mathrm{J}$, is defined as:

$$
\mathrm{v}^{*}\left(\mathrm{x}, \mathrm{a}^{*}\right)=\sup _{\mathrm{a} \in \mathrm{A}}[\mathrm{J}(\mathrm{x}, \mathrm{a})]
$$

It can be obtained by solving a DPE (dynamic programming equation):

$$
\mathrm{v}^{*}=\mathrm{Tv}^{*} \text {, }
$$

where $\mathrm{T}$ is a contraction mapping and:

$$
\operatorname{Tv}(x)=\max _{a \in A}\left[r(x, a)+\beta \sum_{j=1}^{N} v(x) p_{i, j}^{a}\right]
$$

The unique optimal solution of the above DPE can be calculated iteratively by the successive approximation method [1]:

$$
v_{n}(x)=\max _{a \in A}\left[r(x, a)+\beta \sum_{j=1}^{N} v_{n-1}(x) p_{i, j}^{a}\right]
$$

Therefore, for a specific control problem, once the transition matrix and the reward matrix are defined, then by maximizing the total expected reward, a policy for choosing an alternative for each state can be obtained. This represents the optimal strategy which should be taken.

In the following sections, an approach based on the above Markov decision theory is proposed and applied to traffic signal control problem.

\section{ADAPTIVE TRAFFIC SIGNAL CONTROL ALGORITHM}

A typical four-legged intersection is shown in Fig. 1. There are eight movements in this intersection, including four through movements and the four corresponding left turn movements. The number on each movement is labeled according to NEMA (National Electrical Manufacturers Association) convention.

A state space $X$ and a probability measure $P$ must be defined in order to apply the above Markovian decision theory to traffic systems. Since the queue length is the state variable in the traffic dynamics equation, one may want to choose the number of vehicles to be the state of the Markov control model. However, the resulting total number of states is very large. For example, if the number of vehicles under consideration is 20 (per movement), then for an isolated intersection with 8 movements, the number of states is $20^{8} \approx 2.56 \times 10^{10}$, resulting an excessively large matrix dimension. In order to reduce both the computational time and memory space, a way must be found to reduce the number of states. A threshold (number of vehicles) is chosen for the queue of each movement at an intersection. If the queue length of a specific movement is greater than the threshold value, then this movement is defined in the congested mode; otherwise it is in the non-congested mode. These two modes (congestion/non-congestion) are defined as the two states in the binary state space $X$.

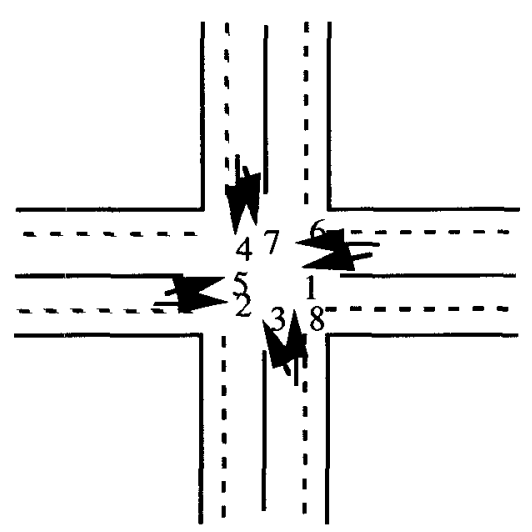

Figure 1. A typical traffic intersection

The state space is discrete, thus the probability measure $\mathrm{P}$ defines a discrete transition law. In the traffic control problem, the probability matrix $\underline{P}$ is time-varying due to the timevarying traffic flow, therefore:

$$
\underline{\mathrm{P}}(\mathrm{k})=\underline{\mathrm{f}_{\mathrm{p}}}\left[\underline{\mathrm{q}}(\mathrm{k}), \underline{\hat{\mathrm{q}}_{\text {in }}}(\mathrm{k}+1), \underline{\mathrm{u}}(\mathrm{k}), \mathrm{q}_{\mathrm{g}}\right]
$$

where $\mathrm{q}(\mathrm{k})$ is the current queue, $\Delta \hat{\mathrm{q}}(\mathrm{k}+1)$ is the estimated number of arrivals in the next time interval, and $\underline{u}(\mathrm{k})$ is the control signal. The probability matrix can be further specified based on different arrival patterns. Under most circumstances, the arrival of vehicles at an isolated intersection follows the Poisson distribution, i.e.:

$$
\mathrm{p}(\mathrm{n})=\frac{(\lambda \Delta \mathrm{t})^{\mathrm{n}} \mathrm{e}^{-\lambda \Delta \mathrm{t}}}{\mathrm{n} !}
$$


where $\mathrm{n}=1,2, \ldots ; \lambda$ is the arrival rate and $\Delta t$ is the time interval. Assuming that at a specific time instant, the current queue length of a specific movement $i$ is denoted by $q_{0}$, and there are $q_{g}$ vehicles passing through the intersection if the signal of this direction is green, then:

$$
\begin{aligned}
& p_{S_{i} \rightarrow N_{i}}^{u_{i}}=p\left(\hat{q}_{i n}^{i}+q^{i}-\delta\left(u_{i}\right) q_{g}^{i} \leq q_{\text {threshold }}^{i}\right) \\
& \text { and } \\
& p_{S_{i} \rightarrow C_{i}}^{u_{i}}=1-p_{S_{i} \rightarrow N_{i}}^{u_{i}}
\end{aligned}
$$

where

$$
\delta\left(u_{i}\right)=\left\{\begin{array}{l}
1, \text { when } u_{i}=G_{i} \\
0, \text { otherwise }
\end{array}\right.
$$

and $S_{i}=N_{i}, C_{i}$ is the current state $\left(N_{i}\right.$ for non-congestion and $\mathrm{C}_{\mathrm{i}}$ for congestion); $u_{i}=G_{i}, R_{i}$ is the control signal $\left(G_{i}\right.$ for green signal and $R_{i}$ for red signal). Two special cases are noted that:

$$
\mathrm{p}_{\mathrm{C}_{\mathrm{i}} \rightarrow \mathrm{C}_{\mathrm{i}}}^{\mathrm{R}_{\mathrm{i}}}=1 \text {, and } \mathrm{p}_{\mathrm{C}_{\mathrm{i}} \rightarrow \mathrm{N}_{\mathrm{i}}}^{\mathrm{R}_{\mathrm{i}}}=0 \text {. }
$$

The reward matrix $\mathrm{R}$ has the same dimension and a definition similar to that of the probability matrix. The control objective herein is to minimize the queue length, so the functions of queue length corresponding to different states are chosen to generate the reward matrix:

$$
\mathrm{R}_{\text {statel, state } 2}^{\mathrm{u}_{\mathrm{i}}}=\mathrm{f}_{\mathrm{u}}\left(\mathrm{q}_{0}^{\mathrm{i}}, \mathrm{q}_{\text {threshold }}^{\mathrm{i}}, \mathrm{u}_{\mathrm{i}}\right)
$$

The signal phasing can be considered as different alternatives for each state. If there are 8 independent movements under 8-phase signal control, the traffic control problem can be formatted as a 256-state Markov process with 8 alternatives for each state. A standard dual-ring control chart with NEMA phases is shown in Fig. 2, where the 8 phases are divided into two groups (rings) by a barrier. In each ring, 4 movements ( 2 through movements and their corresponding left turn movements) must be served if there is demand. Theoretically, there are $2 \cdot 4 !=48$ different phase sequences available, but in fact, in order to avoid conflict traffic, only certain sequences (10 out of 48) are allowed.

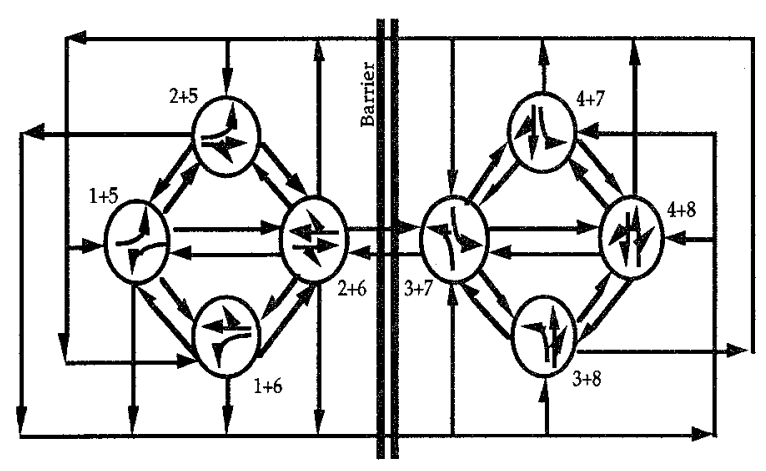

Fig. 2. 8-phase dual ring control

To enforce the phase constraints, a step-by-step decision-making procedure (also termed a "decision tree") is considered. For example, a decision is made first to determine which ring will be served by the Markovian decision algorithm. After this is determined, the second decision is to choose one of the four alternatives from the first decision, again using Markovian decision algorithm. The next phase is either fixed or chosen from the two phases left, depending upon the second decision. At the last decision step for this ring, there is either no phase or just one fixed phase left. This procedure not only guarantees the phase constraints but also dramatically reduces the computation time.

A computational flow chart for traffic intersection control is shown in Fig. 3. To achieve real-time adaptive control for a traffic system, future arrival information is needed. Also, being related to the current state of each traffic movement, the probability matrix and reward matrix are both time-varying variables. However, it is very difficult to make a long term estimation due to the randomness of traffic. Thus the sampling frequency of the traffic system should be set as high as possible. On the other hand, a large sampling rate will increase the cost and computation time. Here the minimum time interval is chosen as $\Delta t=\tau_{\min }$ (i.e., minimum green extension time). Every $\Delta t$ seconds, the time-varying probability matrix $\mathrm{P}$ and the reward matrix are calculated; then a decision is made to choose the control signal for the next time interval based on the current measurement from the 
detector and our estimation. Once the optimal policy is found, it is only implemented for one time step (i.e., $\Delta t$ seconds). At the next time interval, the probability matrix and reward matrix are updated and the whole decisionmaking process is repeated. Therefore, the problem of choosing appropriate signal phasing at a traffic intersection becomes a decisionmaking problem for a Markov process.

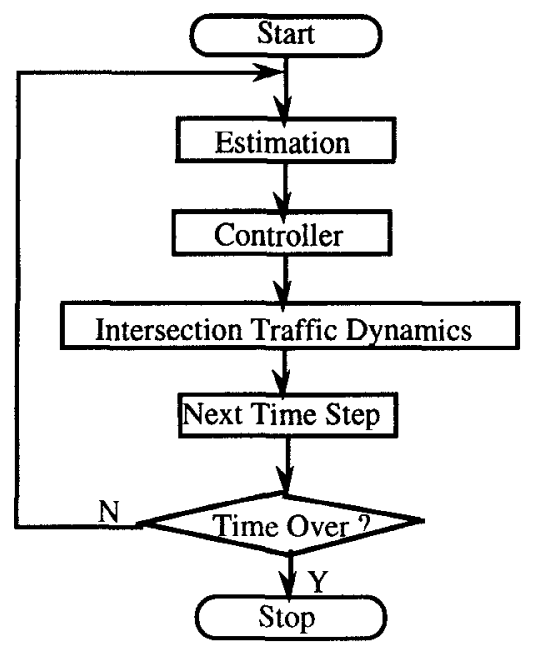

Fig. 3. Flow chart for intersection control

\section{SIMULATION RESULTS}

A simulation of the proposed Markovian adaptive control algorithm applied to an isolated intersection (with a Poisson arrival pattern generated as the external input) was performed to evaluate its performance compared to a fullyactuated control method. Two different cases were considered.

Case I: Uniform (balanced) demand among all conflicting movements.

In this case, the traffic demands on all movements of the intersection are equal. The two algorithms were tested on four different arrival rates, i.e., 200 vehicles per hour per movement, 300 vehicles per hour per movement, 400 vehicles per hour per movement, 500 vehicles per hour per movement, and 600 vehicles per hour per movement. For each arrival rate, the algorithms were tested on forty different sets of random data. The means of the average steady state delay (of the 40 sets of data) were calculated and are listed in table 1, where "MAC" stands for the Markov adaptive control algorithm, and "FAC" stands for the fullyactuated control. The percentage of improvement can also be found in the table 1 . Fig. 4 shows the means (of the 40 sets of data) of the steady state delay of the two different algorithms, where the solid line represents the Markov algorithm and the dotted line represents the fully actuated control.

\begin{tabular}{|c|c|c|c|}
\hline $\begin{array}{c}\text { Arrival } \\
\text { rate }\end{array}$ & FAC & MAC & $\begin{array}{c}\text { Improve- } \\
\text { ment (\%) }\end{array}$ \\
\hline 200 & 11.25 & 11.51 & $-2.31 \%$ \\
\hline 300 & 16.61 & 12.26 & $26.19 \%$ \\
\hline 400 & 29.60 & 13.34 & $54.93 \%$ \\
\hline 500 & 41.64 & 18.27 & $56.12 \%$ \\
\hline 600 & 68.09 & 53.73 & $21.09 \%$ \\
\hline
\end{tabular}

Table 1. Comparison of two algorithms (Case 1)

From distribution-free order statistics analysis, the upper and lower limits within which at least $90 \%$ of the probability of the steady state delay lies with $92 \%$ confidence are plotted in Figure 5:

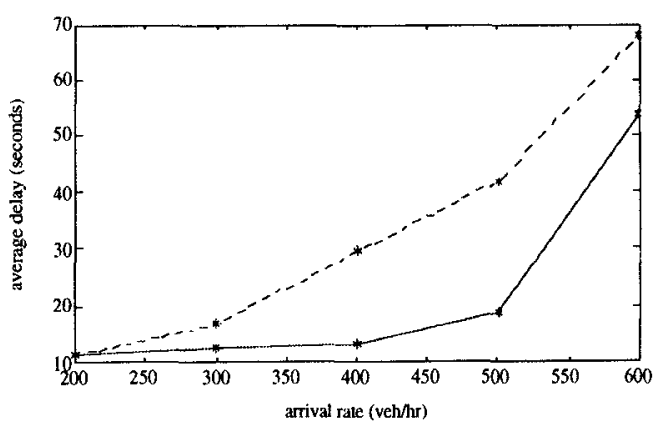

Figure 4. Comparison of two algorithms (Case 1) 


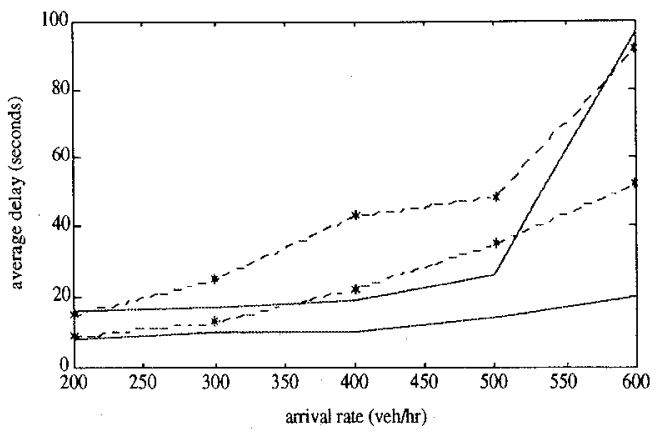

Figure 5. Bounds for simulation results (Case 1)

When the traffic volume is slight (e.g., arrival rate is 200 vehicles/hour/movement), the performance of Markov algorithm is comparable with the fully actuated controller. However, when the traffic volume increases, the Markov algorithm outperforms the traditional one. For example, when $\lambda=300$, the Markov algorithm shows a $26.19 \%$ improvement on the average steady state delay. When $\lambda=400$ and $\lambda=500$, the average steady state delay of the Markov controller is about one half of that of the fully-actuated controller. When $\lambda$ is further increased, the intersection becomes saturated, resulting in large delays for both algorithms.

Case II: Through traffic demand dominates the left-turn demand.

In this case, the ratio of the demands of the through traffic and the left-turn traffic is $2: 1$. This is the more common case compared to the previous one. Similar to case 1 , the two algorithms were also tested on four different conditions, as listed in table 2. For each situation, the algorithms were tested on fifteen different sets of random data. The means (of the 15 sets of data) of the average steady state delay of the two different algorithms, with percentage of improvement, were calculated and are listed in table 3 . The mean and upperlower bounds of data are also plotted in Fig. 6 and Fig. 7, where again the solid line represents the Markov algorithm and the dotted line represents the fully actuated control.

\begin{tabular}{|c|c|c|c|c|c|}
\hline Phase & Test 1 & Test 2 & Test 3 & Test 4 & Test 5 \\
\hline 1 & 250 & 300 & 350 & 400 & 450 \\
\hline 2 & 500 & 600 & 700 & 800 & 900 \\
\hline 3 & 250 & 300 & 350 & 400 & 450 \\
\hline 4 & 500 & 600 & 700 & 800 & 900 \\
\hline 5 & 250 & 300 & 350 & 400 & 450 \\
\hline 6 & 500 & 600 & 700 & 800 & 900 \\
\hline 7 & 250 & 300 & 350 & 400 & 450 \\
\hline 8 & 500 & 600 & 700 & 800 & 900 \\
\hline
\end{tabular}

Table 2. Arrival rates (VPH) for testing

\begin{tabular}{|c|c|c|c|}
\hline & FAC & MAC & $\begin{array}{l}\text { Improve- } \\
\text { ment (\%) }\end{array}$ \\
\hline Test 1 & 25.01 & 17.52 & $29.95 \%$ \\
\hline Test 2 & 32.31 & 20.58 & $36.30 \%$ \\
\hline Test 3 & 49.45 & 26.16 & $51.24 \%$ \\
\hline Test 4 & 116.24 & 62.81 & $45.97 \%$ \\
\hline Test 5 & 166.50 & 129.26 & $22.37 \%$ \\
\hline
\end{tabular}

Table 3. Comparison of two algorithms (Case 2)

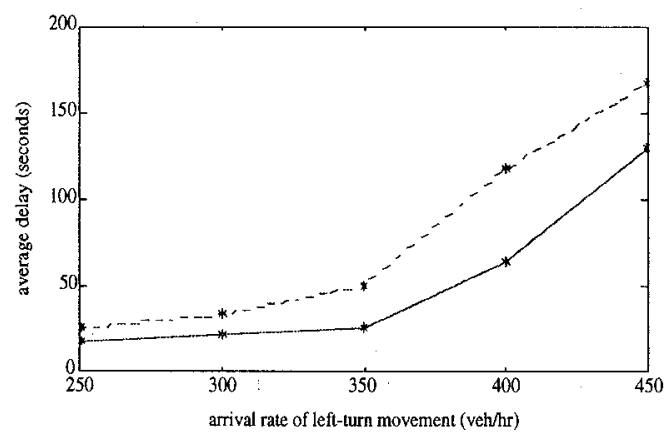

Figure 6. Comparison of two algorithms (Case 2) 


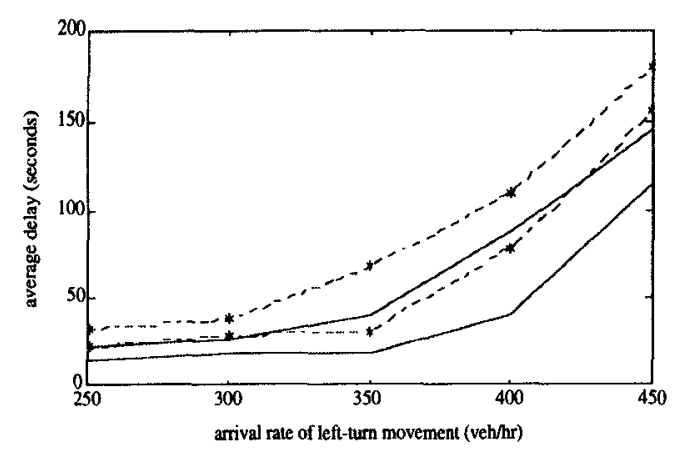

Figure 7. Bounds for simulation results (Case 2)

The simulation results indicate that by applying the Markov adaptive control algorithm, the average delay of intersection can be reduced dramatically ( $22 \%$ to $51 \%)$.

\section{CONCLUSIONS}

In this paper, an optimal strategy for traffic signal control based on Markov decision theory is presented. Computer simulation results indicate that this new approach is more efficient than the traditional fully-actuated control, especially under the conditions of high, but not saturated, traffic demand. Further evaluation and testing will be performed.

\section{ACKNOWLEDGMENT}

This research work was supported by the California Department of Transportation, under its Advanced Transportation Management Systems Testbed Program.

\section{REFERENCES}

[1] Hernandez-Lerma, O., Adaptive Markov control process, Applied mathematical sciences 79, Springer-Verlag, 1989
[2] Recker, W., Ramanathan, B., Yu, X.-H., et. al., "Markovian real-time adaptive control of signal systems", Journal of mathematical and computer modeling, Pergamon Press, vol. 22, August 1995

[3] McShane, W., Roess, R., Traffic Engineering, Prentice-Hall, 1990

[4] Wilshire, R., Black, R., et al., Traffic control systems handbook, FHWA-IP-85-12, 1985 